\title{
On four-dimensional anti-self-dual gradient Ricci solitons
}

\author{
Xiuxiong Chen and Yuanqi Wang
}

\begin{abstract}
In this note we prove that any four-dimensional half conformally flat gradient steady Ricci soliton must be either Bryant's soliton or Ricci flat. We also classify four-dimensional half conformally flat gradient shrinking Ricci solitons with bounded curvature.
\end{abstract}

\section{Introduction.}

In 1982, R.Hamilton introduced the Ricci flow in [10], where he deforms a Riemannian metric in the direction of its Ricci tensor multiplied by -2

$$
\frac{\partial g_{i j}}{\partial t}=-2 R i c_{i j}
$$

The Ricci flow equation gives a canonical way of deforming an arbitrary metric to a critical metric (an Einstein metric in particular). It has been remarkably successful program over years, in particular, the seminal work of G. Perelman on the Poincare conjecture. More recently, S. Brendle and R. Schoen prove the Differentiable Sphere Theorem for pointwise 1/4-pinched manifolds (cf. [4]). Note the classical sphere theorem need to assume a global $1 / 4$ pinched condition on curvature.

In this paper, we will study the Ricci solitons. Following R. Hamilton [12], a smooth complete Riemannian manifold $(M, g)$ is called a gradient Ricci soliton if there exists a smooth function $f$ on $M$ and a constant $\rho$ such that the following identity holds:

$$
R_{i j}+\nabla_{i} \nabla_{j} f=\rho g .
$$

We say it's shrinking if $\rho>0$, steady if $\rho=0$ or expanding if $\rho<0$.

Gradient Ricci solitons are extensions of Einstein metrics and it plays an important role in the singularity analysis of Hamilton's Ricci flow.

In the 2-dim case, Hamilton [11] discovered the cigar soliton, which is a complete noncompact gradient steady soliton on $\mathbb{R}^{2}$. The metric is

$$
g_{\text {cigar }}=\frac{d x \otimes d x+d y \otimes d y}{1+x^{2}+y^{2}} .
$$

This soliton is positively curved and looks like a cylinder of finite circumference at infinity. Furthermore, Hamilton [11] showed that the only complete steady soliton on a 2-dimensional manifold with bounded scalar curvature $R$ which attains its maximum at a point is the cigar soliton up to a scaling. 
For $n \geq 3$, R.Bryant showed in [5] that there exists an unique complete rotationally symmetric gradient Ricci soliton on $\mathbb{R}^{n}$ up to scaling. The Bryant soliton has positive curvature operator, and curvature tensor decays in the order of $\frac{1}{r}$.

In 3-dimensional case, Perelman claimed in [19] without proof that the Bryant soliton is the only 3dim complete noncompact positively curved $\kappa$-noncollapsed gradient steady soliton. It's important to understand the picture hidden behind. On this direction there are a lot of interesting works, for example the works of S.Brendle [3], B.Chen [8] and Cao-Cheng [6]. For $n \geq 4$, assuming the Weyl-tensor vanishes, Cao and Chen proved in [6] that for $n \geq 4$, any $n$-dimensional noncompact gradient steady Ricci soliton with vanishing Weyl-tensor and positive curvature operator is isometric to the Bryant soliton (up to a scaling).

The classification of the complete gradient shrinking solitons is also important since they are the blown up limits of type-I singularities of compact Ricci flows (see [17]) and blown down limits of ancient solutions of certain types(see Perelman [19]). On this direction there are a lot of interesting works, for example the works of T.Ivey [13], Perelman [20], Naber[17], Ni-Wallach[18], Gu-Zhu[9], Kotschwar[14], Peterson-Wylie[21].

The problem we consider is the classification of 4-dimensional half conformally flat steady and shrinking gradient Ricci solitons. Half conformally flat metrics are also known as self-dual or antiself-dual if $W^{-}=0$ or $W^{+}=0$, respectively. Without loss of generality, we can assume the soliton is anti-self-dual. Our main results are :

Theorem 1.1. Any 4-dim complete gradient steady Ricci soliton with $W^{+}=0$ must be isometric to one of the following two types:

\section{The Bryant Soliton (up to a scaling).}

2. A manifold which is anti-self-dual and Ricci flat.

Theorem 1.2. Any 4-dim complete gradient shrinking Ricci soliton with bounded curvature and $W^{+}=$ 0 must be isometric to finite quotients of $R^{4}, S^{3} \times R, S^{4}$ or $C P^{2}$.

Remark 1.3. Obviously if we change the condition $W^{+}=0$ to $W^{-}=0$, the conclusions are exactly the same. The proofs of Theorem 1.1 and 1.2 both originate from a wonderful idea in Cao-Chen [6]. On Theorem 1.1, note that we don't require the curvature to be bounded globally. In case (2) a complete picture is still lacking, one can see Remark 2.8 .

On Theorem 1.2, the reason we need the curvature to be uniformly bounded is that we want the metric to be analytic. The fact that Ricci flows with uniformly bounded curvature are analytic when $t>0$ is proved by Bando in [1]. We strongly conjecture that Ricci Solitons should all be analytic without any curvature condition.

Notice that Theorem 1.2 implies that the Kähler shrinking Ricci soliton on $C P^{2} \sharp \overline{C P^{2}}$ ( found by Koiso [15] and Cao [7]), and the soliton on $C P^{2} \sharp 2 \overline{C P^{2}}$ ( found by Wang and Zhu [25]) are not either anti-selfdual or self-dual.

Acknowledgements: Both authors are grateful to Bing Wang for observing Lemma 2.6 and lots of helpful discussions. The second author wants to thank Prof Gang Tian for helpful suggestions. The second author also benefits a lot from communication with Prof Xiaohua Zhu. 


\section{Proof of Theorem 1.1 .}

From now on we assume $(M, g)$ is anti-self-dual, i.e for some corrrect oriented orthonomal basis $e_{1}, e_{2}, e_{3}, e_{4}$ we have

$$
W_{12 k l}^{-}=-W_{34 k l}^{-}, W_{13 k l}^{-}=-W_{42 k l}^{-}, W_{14 k l}^{-}=-W_{23 k l}^{-}
$$

In this section we assume that $(M, g, f)$ satisfies $R_{i j}+\nabla_{i} \nabla_{j} f=0$. Our crucial observation is that for Ricci solitons, the condition $W^{+}=0$ actually implies that the full Weyl tensor vanishes at those points where $\nabla f \neq 0$.

Lemma 2.1. Suppose $(M, g)$ is an anti-self-dual steady Ricci soliton. Suppose $q \in M$ is not a critical point of $f$ i.e $|\nabla f|(q) \neq 0$. Then at the point $q, e_{1}=\nabla f /|\nabla f|$ is an eigenvector of Ric. Furthermore, there exists two constants $\lambda$ and $\mu$ such that for any orthonormal basis $e_{2}, e_{3}, e_{4}$ tangent to the level surface of $f$ at $p$, the Ricci tensor has the following properties:

1. $\operatorname{Ric}\left(e_{1}, e_{1}\right)=\lambda$,

2. $\operatorname{Ric}\left(e_{i}, e_{j}\right)=\mu \delta_{i j}, i, j=2,3,4$.

3. $\operatorname{Ric}\left(e_{1}, e_{j}\right)=R_{1 j}=0, j=2,3,4$,

Proof. of lemma 2.1:

Let $n=4$. First we recall the definition of the Cutton tensor:

$$
C_{i j k}=-\frac{n-3}{n-2} W_{i j k l, l}
$$

and

$$
C_{i j k}=R_{j k, i}-R_{i k, j}-\frac{1}{2(n-1)}\left(R_{i} g_{j k}-R_{j} g_{i k}\right)
$$

Using $R_{j k}+f_{j k}=0$, we have

$$
\begin{aligned}
& C_{i j k}=f_{i k j}-f_{j k i}-\frac{1}{2(n-1)}\left(R_{i} g_{j k}-R_{j} g_{i k}\right) \\
& =-R_{i j k l} f_{l}-\frac{1}{2(n-1)}\left(R_{i} g_{j k}-R_{j} g_{i k}\right)
\end{aligned}
$$

Using

$$
\begin{aligned}
& R_{i j k l} \\
= & W_{i j k l}+\frac{1}{n-2}\left(R_{i k} g_{j l}+R_{j l} g_{i k}-R_{i l} g_{j k}-R_{j k} g_{i l}\right) \\
& -\frac{R}{(n-1)(n-2)}\left(g_{j l} g_{i k}-g_{i l} g_{j k}\right)
\end{aligned}
$$


We get

$$
\begin{aligned}
& C_{i j k}=-W_{i j k l} f_{l}-\frac{1}{n-2}\left(R_{i k} g_{j l}+R_{j l} g_{i k}-R_{i l} g_{j k}-R_{j k} g_{i l}\right) f_{l} \\
+ & \frac{R}{(n-1)(n-2)}\left(g_{j l} g_{i k}-g_{i l} g_{j k}\right) f_{l}-\frac{1}{2(n-1)}\left(R_{i} g_{j k}-R_{j} g_{i k}\right) \\
= & -W_{i j k l} f_{l}-\frac{1}{n-2}\left(R_{i k} f_{j}-R_{j k} f_{i}\right)+\frac{1}{2(n-2)}\left(g_{j k} R_{i}-g_{i k} R_{j}\right) \\
+ & \frac{R}{(n-1)(n-2)}\left(f_{j} g_{i k}-f_{i} g_{j k}\right)-\frac{1}{2(n-1)}\left(R_{i} g_{j k}-R_{j} g_{i k}\right)
\end{aligned}
$$

Using $2 R_{j l} f_{l}=R_{j}$, we get

$$
\begin{aligned}
& C_{i j k} \\
= & -W_{i j k l} f_{l}-\frac{1}{n-2}\left(R_{i k} f_{j}-R_{j k} f_{i}\right)+\frac{1}{(n-2)(n-1)}\left(g_{j k} R_{i l} f_{l}-g_{i k} R_{j l} f_{l}\right) \\
+ & \frac{R}{(n-1)(n-2)}\left(f_{j} g_{i k}-f_{i} g_{j k}\right)
\end{aligned}
$$

Since $\left.\nabla f\right|_{p} \neq 0$, suppose $f(p)=c$. We denote

$$
\begin{aligned}
& B_{i j k} \\
= & -\frac{1}{n-2}\left(R_{i k} f_{j}-R_{j k} f_{i}\right)+\frac{1}{(n-2)(n-1)}\left(g_{j k} R_{i l} f_{l}-g_{i k} R_{j l} f_{l}\right) \\
+ & \frac{R}{(n-1)(n-2)}\left(f_{j} g_{i k}-f_{i} g_{j k}\right)
\end{aligned}
$$

Choose $e_{1}, e_{2}, e_{3}, e_{4}$ which diagonalize Ric at $q$. Assuming $W^{+}=0$, we get that:

$$
\begin{aligned}
& B_{12 k}+B_{34 k} \\
= & C_{12 k}+C_{34 k}+\left(W_{12 k l}+W_{34 k l}\right) f_{l} \\
= & -\frac{n-3}{n-2}\left(W_{12 k l}+W_{34 k l}\right)_{, l}+\left(W_{12 k l}+W_{34 k l}\right) f_{l} \\
= & 0
\end{aligned}
$$

Similarly we also have $B_{13 k}+B_{42 k}=0, B_{14 k}+B_{23 k}=0$. By definition we see that $B_{i j k}=0$ if $i, j, k$ are mutually different. So we have:

$$
B_{i j i} \equiv 0, \text { for all } i, j=1,2,3,4 \text {. }
$$

Furthermore by definition of $B$ again we have $B_{i i j}=0$. Then we see that $B \equiv 0$.

Now we denote the eigenvalue of Ric with respect to $e_{k}, k=1,2,3,4$ to be $\lambda_{k}, k=1,2,3,4$. respectively.

Now let $n=4$, take $B_{121}$ as an example, we have

$$
0=-\frac{1}{2}\left(\lambda_{1} f_{2}\right)-\frac{1}{6} f_{2} \lambda_{2}+\frac{R}{6} f_{2}=\frac{f_{2}}{6}\left(R-3 \lambda_{1}-\lambda_{2}\right)
$$


Which means we should have

$$
\frac{f_{i}}{6}\left(R-3 \lambda_{j}-\lambda_{i}\right)=0, \text { for all } i \neq j
$$

Thus if $\nabla f$ has more than two nonzero component, we have $\lambda_{1}=\lambda_{2}=\lambda_{3}=\lambda_{4}$. If $\nabla f$ has only one nonzero component, say $f_{1}$, then $\lambda_{2}=\lambda_{3}=\lambda_{4}$. In either case, $\nabla f$ is an eigenvector of Ric and $\left.\operatorname{Ric}\right|_{(\nabla f)^{\perp}}=\mu \delta a b$.

Next let $K_{c}=\{f(p)=c\}$ be the level set of $f$. The proof of the next lemma is essentially the same as lemma 3.3 in [6].

Lemma 2.2. Suppose $(M, g)$ is an anti-self-dual steady gradient Ricci soliton. Suppose $q \in M$ is not a critical point of $f$ i.e $|\nabla f|(q) \neq 0$. Then near the point $p$ we have that the second fundamental form $\Pi_{i j}$ of $K_{c}$ is a constant multiple of the of the metric i.e $\Pi_{i j}=\frac{\eta}{3} g_{i j}$, in which $\eta$ is the mean curvature. Furthermore, $\eta$ is constant on $K_{c}$.

Proof. of Lemma2.2.

From Lemma 2.1 we can set $e_{1}=\frac{\nabla f}{|\nabla f|}$. First notice that $\nabla f \neq 0$ at $q$ implies that there exists a coordinate system $\psi_{q}$ near $q$ such that $f$ is one of coordinate functions of $\psi_{q}$. Suppose $i, j \neq 1$, restricted to the distribution $\nabla f^{\perp}$, we compute

$$
\Pi_{i j}=\frac{\nabla \nabla f}{|\nabla f|}=\frac{-R_{i j}}{|\nabla f|}=\frac{-\frac{R-R_{11}}{3}}{|\nabla f|} g_{i j}
$$

Thus the mean curvature satisfies $\eta=\frac{-R+R_{11}}{|\nabla f|}$.

Next we show that $\eta$ is a constant along fiber direction. Since 2.1 holds, then direct computation shows that for $i, j, k=2,3,4$ we have

$$
R_{1 i j k}=-\nabla_{j}^{T} \Pi_{k i}+\nabla_{k}^{T} \Pi_{j i}
$$

Contracting $i$ and $k$, we obtain

$$
0=R_{1 j}=-\nabla_{j}^{T} \eta+\nabla_{k}^{T} \Pi_{j k}=-\frac{2}{3} \nabla_{j}^{T} \eta
$$

Since $\mathrm{j}$ is arbitrary, $\eta$ is constant over $K_{c}$.

Lemma 2.3. Let $(M, g, f)$ be a 4-dim steady Ricci soliton and $W^{+}=0$. Suppose $q \in M$ and $|\nabla f|^{2}(q) \neq$ 0 , then $W=0$ at $q$.

Proof. of Lemma2.3.

From the proof of lemma 2.2 we have known what $R_{1 i j k} \equiv 0$ at $q$, if $\{i, j, k\}=\{2,3,4\}$. Recall we have the curvature decomposition:

$$
R_{i j k l}=\frac{1}{2}\left[A_{i k} g_{j l}+g_{i k} A_{j l}-A_{i l} g_{j k}-g_{i l} A_{j k}\right]+W_{i j k l} .
$$


The $A$ is the Schouten tensor:

$$
A=\frac{2}{n-2}\left(R_{i j}-\frac{R}{2 n-2} g_{i j}\right)
$$

Since $e_{1}, e_{2}, e_{3}, e_{4}$ are all eigenvectors of Ric, then they are also eigenvectors of $A$. Then we have

$$
(A \otimes g)_{1 a b c}=0, \text { for all } a, b, c=\{2,3,4\} .
$$

Thus from 2.2 and our assumption that $W^{+}=0$, we have:

$$
W_{1 a b c}^{-}=0, \text { for all } a, b, c=\{2,3,4\} \text {. }
$$

A well known property of $W^{-}$says that for any $k, l=1,2,3,4$, we have

$$
W_{12 k l}^{-}=-W_{34 k l}^{-}, W_{13 k l}^{-}=-W_{42 k l}^{-}, W_{14 k l}^{-}=-W_{23 k l}^{-}
$$

Thus combining 2.3 and 2.4 we have

$$
W^{-} \equiv 0,
$$

which means $g$ actually has vanishing Weyl tensor at $q$.

Next we should quote two statements which are very important to us.

Theorem 2.4. (Cao-Chen[6]) Suppose $n \geq 4$. Any complete $n$-dim gradient steady soliton with vanishing Weyl tensor must be either flat or isometric to the Bryant soliton.

Proposition 2.5. (B.L.Chen[8]) Any complete ancient solution to the Ricci flow has nonnegative scalar curvature everywhere.

Based on Proposition 2.5, Bing Wang has an observation.

Lemma 2.6. (Bing Wang [24]) Suppose $(M, g, f)$ is a gradient steady Ricci soliton i.e:

$$
\text { Ric }+\nabla \nabla f=0 .
$$

If $|\nabla f|^{2}=0$ over an open set, then $f \equiv$ constant over the whole manifold $M$ and $g$ is Ricci flat.

Proof. of Lemma2.6.

Suppose $|\nabla f|^{2}$ vanishes in a ball $B_{v}$. By definition of steady soliton, we have $R \equiv 0$ in the $B_{v}$. Since $R+|\nabla f|^{2}=$ Constant over the manifold $M$, then over $B_{v}$ we obviously have $C=0$. Thus

$$
R+|\nabla f|^{2}=0 \text { over the manifold } \mathrm{M} \text {. }
$$

But according to B.Chen's result 2.5, any steady Ricci soliton (not necessarily with bounded curvature) has $R \geq 0$, then $R \equiv 0 \equiv|\nabla f|^{2}$. Which means $f \equiv C_{1}$ and $(M, g)$ is Einstein.

Now we are ready to prove the first main theorem. 
Proof. of Theorem 1.1 .

First we assume the set $\left\{|\nabla f|^{2} \neq 0\right\}$ is dense in $M$, then according to lemma 2.3, $W=0$ over a dense set. By continuity of the Weyl tensor we have $W=0$ everywhere. By Cao-Chen's classification 2.4, we are in case (1) or (2) in Theorem 1.1 .

If the above does not hold, then $|\nabla f|^{2}$ vanishes in a ball $B_{v}$. By lemma $2.6 f$ is a constant over $M$ and $g$ is Ricci flat. Therefore we are in case (2) of Theorem 1.1.

Remark 2.7. One should notice that if the curvature is uniformly bounded, the fact Ric $\equiv 0$ over the whole manifold $M$ provided that $|\nabla f|^{2}$ vanishes in a ball is also directly implied by lemma 3.4 .

Remark 2.8. One might be interested in the 2nd case in Theorem 1.1. Actually in the special case that $(M, g)$ is $A L E$ and hyper-Kähler, Kronheimer obtained a very nice classification (see [16]). The Eguchi-Hanson metric is in Kronheimer's classification. Note that hyper-Kählerity implies $(M, g)$ is half conformal flat and Ricci flat. But if $(M, g)$ is not $A L E$, there are still a lot of possibilities. In Tian-Yau [22], it's showed explicitly that there exists a complete noncompact 4-dim Kähler manifold which is Ricci flat and has volume growing in the rate of $r^{\frac{4}{3}}$. Note again Kähler manifolds with zero Ricci curvature is automatically half conformally flat. Furthermore, Tian-Yau (see [22] and [23]) constructed a vast class of complete noncompact Kähler manifolds of finite topological type. On the other hand, M. Anderson, P. Kronheimer and Le Brun constructed such examples with infinite topological type. There should be other constructions which the authors don't know.

\section{Proof of Theorem 1.2.}

Again we assume $(M, g)$ is anti-self-dual. The basic equation is

$$
R i c+\nabla \nabla f=g .
$$

The situation is essentially the same as the proof of Theorem 1.1, the difference is that we have to apply lemma 3.4 thus we need the curvature to be uniformly bounded. First by the same proof of lemma 2.1 we also have:

Lemma 3.1. Suppose $(M, g)$ is an anti-self-dual gradient shrinking Ricci soliton. Suppose $q \in M$ is not a critical point of $f$ i.e $|\nabla f|(q) \neq 0$. Then at the point $q, e_{1}=\nabla f /|\nabla f|$ is an eigenvector of Ric. Furthermore, there exists two constants $\lambda$ and $\mu$ such that for any orthonormal basis $e_{2}, e_{3}, e_{4}$ tangent to the level surface of $f$ at $p$, the Ricci tensor has the following properties:

1. $\operatorname{Ric}\left(e_{1}, e_{1}\right)=\lambda$,

2. $\operatorname{Ric}\left(e_{i}, e_{j}\right)=\mu \delta_{i j}, i, j=2,3,4$.

3. $\operatorname{Ric}\left(e_{1}, e_{j}\right)=R_{1 j}=0, j=2,3,4$,

Therefore we also have the following lemma by the same proof of lemma 2.3 .

Lemma 3.2. Let $(M, g, f)$ be a 4-dim complete gradient shrinking Ricci soliton with bounded curvature and $W^{+}=0$. Suppose $q \in M$ and $|\nabla f|^{2}(q) \neq 0$, then $W=0$ at $q$. 
Next we recall a result of Bando in 3.3 on analyticity of Ricci flows.

Theorem 3.3. (Bando)Suppose $\{M, g(t), t \in[0, T]\}$ is a complete Ricci flow with bounded curvature, then $g(t)$ is analytic in polar coordinates for $t>0$.

Bando's result directly implies the following lemma.

Lemma 3.4. Let $(M, g, f)$ is a complete Ricci soliton with bounded curvature, i.e:

$$
R_{i j}+\nabla_{i} \nabla_{j} f=\rho g_{i j} \text { for some constant } \rho \text { and smooth function } f \text {. }
$$

Suppose there exists an open set $\Omega$ in which $|\nabla f|=0$, then $f$ is a constant over the whole manifold $M$ and $(M, g)$ is Einstein.

Proof. of Lemma 3.4

Obviously we have $f=C_{1}$ in $\Omega, C_{1}$ is a constant. If the lemma does not hold, there exist a short geodesic $\gamma(s), s \in\left[0, s_{0}\right]$ with arclength parameter and a number $v$ such that

$$
f[\gamma(s)]=C_{1} \text {, when } s<v ; f\left[\gamma\left(s_{0}\right)\right] \neq C_{1} .
$$

Notice for all $p \in M$ and any geodesic $\gamma$ passing through $p, \gamma$ is a straight line in the polar coordinate centered at $p$ and therefore analytic. By the bounded curvature assumption and Bando's result, we know that $\operatorname{Ric}(\gamma \dot{(s)}, \gamma(s))$ is analytic in $s$, which means that $f^{\prime \prime}(s)$ is analytic in $s$. Then $f$ is also analytic in $s$ which implies $f(s) \equiv C_{1}$ for all $s \in\left[0, s_{0}\right]$. This is a contradiction.

Before proving Theorem 1.2, we shall quote the main theorem in [26].

Theorem 3.5. (Z-H Zhang)When $n \geq 4$, any complete gradient shrinking soliton with vanishing Weyl tensor must be the finite quotients of $R^{n}, S^{n-1} \times R$, or $S^{n}$.

Now we are ready to prove Theorem 1.2 .

Proof. of Theorem 1.2 .

Similarly, we first assume the set $\left\{|\nabla f|^{2} \neq 0\right\}$ is dense in $M$, then according to lemma2.3, $W=0$ over a dense set. By continuity of the Weyl tensor we have $W=0$ everywhere. By Zhang's classification 2.4 $(M, g)$ must be the finite quotients of $R^{4}, S^{3} \times R$ or $S^{4}$.

If the above does not hold, then $|\nabla f|^{2}$ vanishes in a ball $B_{v}$. By lemma 3.4, we have that Ric $\equiv g$ over the whole manifold $M$. Therefore $M$ is compact Einstein with positive scalar curvature and $W^{+}=0$, then it's necessarily isometric to $S^{4}$ or $C P^{2}$ according to a theorem of Hitchin(see theorem 13.30 of [2]). Thus Theorem 1.2 is true. 


\section{References}

[1] Bando, Shigetoshi. Real analyticity of solutions of Hamilton's equation, Math. Zeit. 195 (1987), 93-97.

[2] Besse, A., Einstein Manifolds. Springer-Verlag, Berlin, 1987. MR0867684 (88f:53087)

[3] S. Brendle, Uniqueness of gradient Ricci solitons. arXiv:1010.3684

[4] S. Brendle, Ricci Flow and the Sphere Theorem, Graduate Studies in Mathematics, vol. 111, American Mathematical Society (2010)

[5] Bryant, R., unpublished work

[6] Cao, H.-D, Chen Qiang. On Locally Conformally Flat gradient Steady Ricci Solitons.arXiv:0909.2833

[7] Cao, H.-D., Existence of gradient Kähler-Ricci solitons, Elliptic and Parabolic Methods in Geometry (Minneapolis, MN, 1994), A K Peters, Wellesley, MA, (1996) 1-16.

[8] Chen, B.-L., Strong uniqueness of the Ricci flow, J. Differential Geom. 82 (2009), 363-382. MR2520796 (2010h:53095)

[9] H.L.Gu and X.P.Zhu, The Existence of Type II Singularities for the Ricci Flow on $S^{n+1}$, Comm. Anal. Geom. Volume 16, Number 3 (2008), 467-494.

[10] Hamilton, R. S.. Three-Manifolds with Positive Ricci Curvature. J. Diff. Geom. 1982.

[11] Hamilton, R. S., The Ricci flow on surfaces, Contemporary Mathematics 71 (1988), 237-261. MR0954419 (89i:53029)

[12] Hamilton, R. S., The formation of singularities in the Ricci flow, Surveys in Differential Geometry (Cambridge, MA, 1993), 2, 7-136, International Press, Combridge, MA, 1995. MR1375255 (97e:53075)

[13] Ivey, T., Ricci solitons on compact three-manifolds, Diff. Geom. Appl. 3 (1993), 301-307. MR1249376 (94j:53048)

[14] B.Kotschwar, On rotationally invariant shrinking gradient Ricci solitons , arXiv:math.DG/0702579.

[15] Koiso, N.,On rotationally symmmetric Hamiltons equation for Kähler-Einstein metrics, Recent Topics in Diff. Anal. Geom., Adv. Studies Pure Math.,18-I,Academic Press, Boston, MA (1990), $327 \mathrm{C} 337$.

[16] P.B.Kronheimer, The construction of ALE spaces as hyper-Kahler quotions, J. Differ. Geom. 29 (1989), 665-683. 
[17] A.Naber, Noncompact Shrinking 4-Solitons with Nonnegative Curvature, arXiv:math.DG/0710.5579.

[18] L.Ni and N.Wallach, On a classification of the gradient shrinking solitons, arXiv:math.DG/0710.3194.

[19] G.Perelman, The entropy formula for the Ricci flow and its geometric applications, arXiv:math. DG/0211159.

[20] G.Perelman, Ricci flow with surgery on three manifolds, arXiv:math. DG/0303109.

[21] P.Petersen and W.Wylie, On the classification of gradient Ricci solitons. arXiv:math.DG/0712.1298.

[22] G. Tian; Shing Tung Yau,Complete Kähler Manifolds with Zero Ricci Curvature. I Journal of the American Mathematical Society, Vol. 3, No. 3. (Jul., 1990), pp. 579-609.

[23] Gang Tian, Shing Tung Yau,Complete Kähler manifolds with zero Ricci curvature II Invent. math. 106, 27-60 (1991).

[24] Bing Wang. private communication.

[25] Wang, X-J and Zhu, X.H. Kahler-Ricci solitons on toric manifolds with positive first Chern class. Advances in Math. 188 (2004) 87-103.

[26] Zhang, Z.-H., Gradient shrinking solitons with vanishing Weyl tensor, Pacific J. Math. 242 (2009), no. 1, 189-200.

Xiuxiong Chen, Department of Mathematics, University of Wisconsin-Madison, Madison, WI 53706, USA; xiu@math.wisc.edu

Yuanqi Wang, Department of Mathematics, University of Wisconsin-Madison, Madison, WI, 53706, USA; wangyuanqi1982@gmail.com 